\title{
DESENVOLVIMENTO DE UM HOMÍMETRO PORTÁTIL E DE BAIXO CUSTO
}

DOI: 10.37702/2175-957X.COBENGE.2021.3393

Stefânia Oliveira Silva - sosilva4@gmail.com

Universidade Federal do Oeste da Bahia

Rua do Machado 81

47600-000 - Bom Jesus da Lapa - BA

João Pedro dos Santos Martins - joaopedrodossantosmartins@yahoo.com.br Universidade Federal do Oeste da Bahia

Rua Manoel Gomes de Souza 127

- Bom Jesus da Lapa - BA

Eclésio dos Santos Rocha - eckles78@gmail.com

Universidade Federal do Oeste da Bahia

Rua Dr. Josefino Moreira de Castro, $1^{\circ}$ Andar 617

47630-000 - SERRA DO RAMALHO - BA

MARCIO ANTONIO DE AZEVEDO - azevedomarcio053@gmail.com

Universidade Federal do oeste da Bahia

Bom Jesus Da Lapa 998

47600-000 - Bom Jesus da Lapa - BA

Resumo: O presente artigo apresenta o desenvolvimento de um ohmímetro microcontrolado portátil e de baixo custo implementado na disciplina de circuitos elétricos I. O trabalho foi iniciado com o intuito de buscar uma solução para atender as demandas práticas em casa, diante da situação do isolamento e impossibilidade de ir aos laboratórios para execução de práticas, dado que a precisão e segurança na aquisição de dados, são algumas das características imprescindíveis no manuseio dos instrumentos digitais para o curso de engenharia elétrica. $O$ desenvolvimento utilizou conteúdos interdisciplinares, partindo do entendimento e principio de funcionamento de um multímetro, a programação de um microcontrolador se deu pelo Arduíno, para o tratamento de dados. Diante das técnicas essenciais e estabelecendo assim, uma prática concisa e objetiva, foi vital e necessário compreender o básico em análise de circuitos, conhecimento em linguagem de programação e componentes físicos fundamentais, como resistores, 
jumpers e protoboard. Além da descrição dos componentes, materiais e métodos utilizados para o desenvolvimento, o trabalho também apresenta alguns testes práticos. Acredita-se que os recursos aqui demonstrados, associados às aulas teóricas, propiciam um ambiente mais favorável à aprendizagem, fazendo com que o aluno tenha curiosidade e prazer em aprender, sendo uma valiosa ferramenta de complementação didática.

Palavras-chave: Arduino, Divisor de tensão, Programação, Resistores 


\section{DESENVOLVIMENTO DE UM HOMÍMETRO PORTÁTIL E DE BAIXO CUSTO}

\section{INTRODUÇÃO}

Falar de educação é uma tarefa que por vezes parece simples, principalmente quando é proposto a análise de aspectos isolados como observar práticas e nada refletir sobre eles. Entretanto, a partir do momento em que há uma reflexão diante do que é observado e praticado, o discurso sobre o aprender se torna mais complexo e desafiador, pois as convicções passam a serem questionadas.

As atividades práticas em sala de aula têm ganhado cada vez mais destaque nas instituições de ensino, com o objetivo de contribuir para a aprendizagem como um todo. A utilização de experimento, como desenvolvimentos de bancadas, e propriamente de equipamentos nas aulas de circuitos elétricos tem se tornado um meio preparatório valioso e enriquecedor. Quando elaborado visando o conhecimento do conteúdo, um experimento tem potencial para tornar-se eficaz ferramenta de discernimento, apresentando grande capacidade para despertar o interesse dos alunos e da comunidade externa pelo conteúdo que se deseja ministrar, principalmente porque abordam conceitos dentro de um ambiente prático e lúdico. Quanto mais as experiências educacionais desenvolvidas pelos discentes se assemelharem às futuras situações, mais fácil torna 0 aprendizado em seus conhecimentos.

E em uma sociedade marcada por ideais da globalização e economia de mercado, é notável a forte presença dos meios tecnológicos para desenvolvimento de equipamentos no qual tem por único e objetivos facilitar o uso em diversas situações. Neste sentido, buscou-se no presente trabalho desenvolver e implementar um ohmímetro que tem como finalidade medir a resistência elétrica de componentes em circuitos eletrônicos. Pretendese que o usuário possa perceber a relação entre teoria e prática ao relacionar dados teóricos com os resultados práticos diante da variação das propriedades intrínsecas dos componentes usados. Propriedades essas que podem causar variações na condutibilidade elétrica dos materiais, variações de valores dos componentes elétricos, formas de medição, e demais fatores físicos, afim de antever as influências e consequências dessas variações no resultado final.

O produto educacional proposto neste artigo busca utilizar a metodologia multidisciplinar como ferramenta no ensino e aprendizagem do curso de engenharia elétrica. A proposta sugerida no presente trabalho utiliza não apenas aulas expositivas e laboratoriais e instrumentos de laboratório controlados, mas também as experiências mais simples, focando mais na busca de conceitos e análise de fenômenos por meio de sistemas de baixo custo.

Visando um produto didático, de precisão e principalmente de baixo custo, o projeto apresenta exigências básicas que um ohmímetro deve oferecer, tendo em vista a aplicação e discernimento do conhecimento apreendido em sala de aula, promovendo assim o desenvolvimento e incentivo à criação de novos projetos que somam os conceitos básicos e necessários apresentados durante a graduação. Para o projeto, a construção do modelo portátil e de baixo custo levou em consideração a precisão das medições a serem obtidas diante dos custos dos componentes utilizados. A partir dos parâmetros determinados, foram necessárias várias pesquisas e estudos afim de construí-lo da forma mais didática possível. 


\section{FUNDAMENTAÇÃO TEÓRICA}

Ao analisar as dificuldades e os problemas enfrentados por estudantes, seja eles ensino médio ou graduação, na aprendizagem de certos conteúdos, e ainda mais em período de pandemia, no qual o acesso às escolas e universidades, em destaque aos laboratórios, ficaram inacessíveis, verifica-se a necessidade de tornar determinados estudos mais concreto e mais próximo da realidade. Devido ao grau de abstração demandado no ensino referente aos conceitos mais complexos em Eletricidade, habilidade ainda em fase de desenvolvimento nos alunos, a aplicação de experimentos em sala de aula evidencia a teoria em exemplos práticos que podem aparecer na rotina dos alunos, como forma de facilitar, motivar e incrementar o aprendizado destes conceitos.

Segundo Gaspar (GASPAR; CASTRO, 2005) é por meio dos experimentos que a ciência encanta e aguça o interesse das pessoas. Assim são as atividades de demonstração, que não necessariamente precisam se restringir às salas de aula, podendo ser manuseadas em outros ambientes, adequando a explicação dos temas a serem apresentados ao público a qual se destina.

E para isso, tem se a metrologia que é a ciência que estuda sob o ponto de vista prático ou teórico os assuntos correspondentes às medições. $O$ instrumento de medição, conforme apresentado na Figura 1, é o responsável por entregar ao usuário o resultado medido. No decorrer do processo o valor real da variável pode diferir do valor medido, e essas diferenças podem ser atribuídas a erros com naturezas distintas que acarreta em uma entrega de dados irreais para o observador.

Nas medições diretas, o instrumento indica o valor mensurado com as mesmas unidades do objetivo a ser medido, ou seja, de maneira natural. Já as medidas realizadas de maneira indireta utilizam-se de uma ou mais grandezas diferentes, para obter a variável objetiva, a exemplo, medir a velocidade média, utilizando as grandezas tempo e espaço (ALBERTAZZI, 2009).

Figura 1 - Instrumento de Medição.

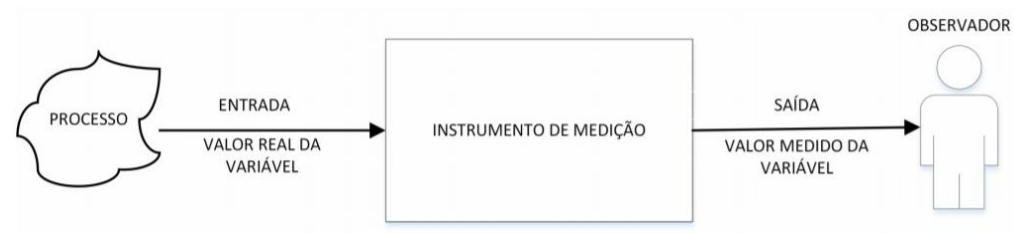

Fonte: DO NASCIMENTO, 2020

A introdução de dispositivos eletrônicos, como circuitos baseados em amplificadores operacionais, tem melhorado muito as características da instrumentação de medidas elétricas. Nomeadamente, a impedância de entrada dos voltímetros aumentou substancialmente, a resistência equivalente do amperímetro também baixou e é possível construir um ohmímetro com relação linear entre a representação do valor e a resistência a medir. Aliando a potencialidade dos circuitos eletrônicos à representação digital dos sinais é possível construir aparelhos de medição eletrônica com elevada precisão, com custos mais baixos e com representação digital de sinal, reduzindo desta forma os erros de leitura.

A representação digital de sinais é constituída por um dispositivo que faz a conversão analógico-digital (A/D) e por um mostrador de sete segmentos. O conversor $A / D$ converte um sinal de tensão proporcional à grandeza que se quer medir o sinal digital. $O$ sinal é processado para ser aplicado a um conjunto de mostradores de sete segmentos que indica o valor da grandeza medida. 


\section{$2.1 \quad$ Resistores}

Nesse contexto, os componentes passivos a exemplos dos resistores, por não possuírem a capacidade de gerar energia, são utilizados em circuitos para modelar 0 comportamento da resistência à corrente de um material (ALEXANDRE, 2013), ou seja, controlam a passagem de corrente pelo circuito, para diminuir a potência do circuito.

Os resistores podem ser construídos em diversos formatos, dos quais são divididos em dois grupos, fixos e variáveis (BOYLESTARD, 2012). Por esse ângulo, os resistores mantêm uma dependência acerca dos materiais a serem utilizados em suas respectivas construções, visto que, a resistência está intrinsecamente relacionada à resistividade do material. A temperatura por sua vez, é um fator ímpar na resistência, pois a mesma pode aumentar à medida que a temperatura se eleva.

Devido alguns resistores serem elementos estritamente pequenos, foi adotado como parâmetros para a identificação de valores, um sistema de código de cores, ilustrado na Figura 2, no qual cada cor representa um respectivo valor.

De acordo com as faixas representadas no resistor, tem-se a primeira e a segunda que representam o primeiro e o segundo digito, respectivamente. $E$ a terceira faixa determina o multiplicador, em potências de dez. A quarta faixa é a tolerância do resistor fornecida pelo fabricante, que é uma indicação da precisão no valor. Entretanto, se o resistor apresentar cinco faixas, as três primeiras são os dígitos, a quarta faixa o algarismo multiplicador e a quinta a tolerância. Caso apareça a sexta faixa deve-se assumir como o coeficiente de temperatura.

Figura 2 - Código de Cores.

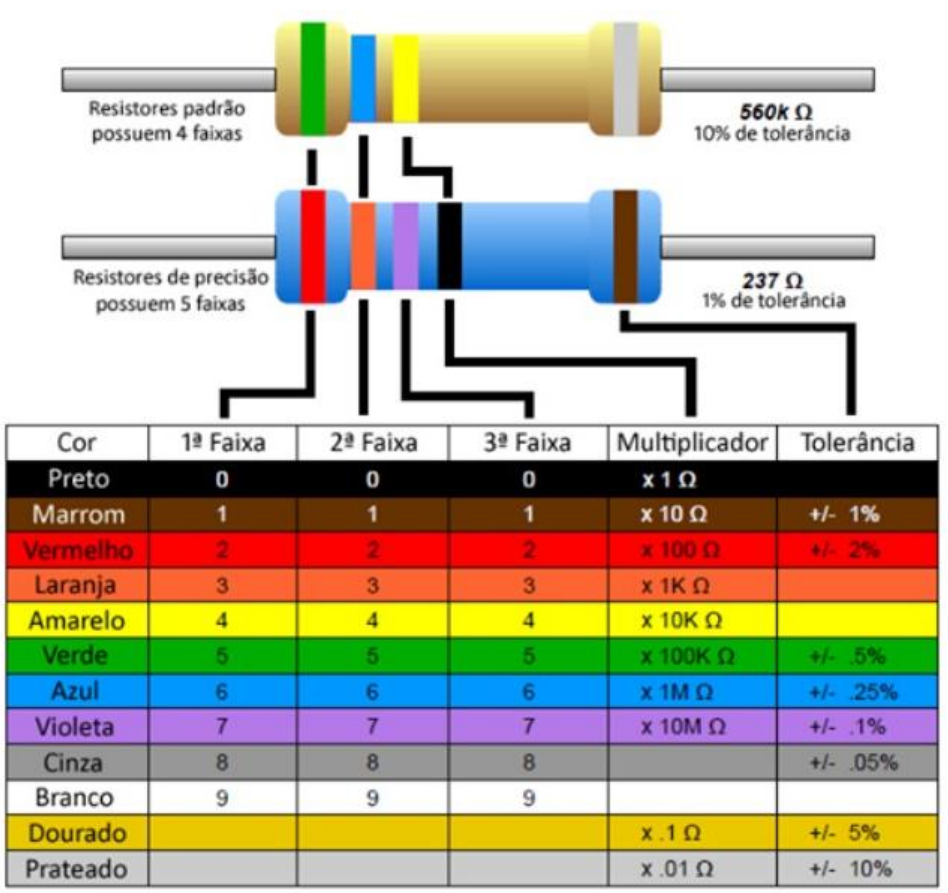

Fonte: BOYLESTAD, 2012.

Atualmente diversos aplicativos e programas gratuitos são encontrados na internet, com o propósito de decifrar os mais variados códigos de cores para os resistores. Com operacionalidade de realizar a conversão analógica/digital e digital/analógico, o Arduino é uma plataforma eletrônica de código aberto baseada em hardware e software fácil de manusear. Mediante a sua versatilidade operacional e diversificação no mercado, a ferramenta vem se destacando no cenário acadêmico com uma infinidade de experimentos, 
desde os mais simples até os mais complexos, desde a demonstração de potencialidade na robótica às análises de dados e medições físicas. Apesar de ser um processo simples, é necessário o mínimo de conhecimento básico em linguagem de programação, visto que, o Arduino opera baseado na linguagem $\mathrm{C}++$.

\section{METODOLOGIA} básicos:

Para o desenvolvimento do projeto do ohmímetro, utilizou-se alguns componentes

- Coletar informações sobre os componentes elétricos utilizados no desenvolvimento do ohmímetro por meio de pesquisa bibliográfica em livros e artigos relacionados, buscando informações específicas referentes a cada componente elétrico;

- Resistores;

- Jumpers;

- Protoboard;

- LEDs;

Para o tratamento e visualização dos dados e das resistências a serem aferidas, usou-se o microprocessador Arduino com display LCD e módulo para sua conexão.

A princípio, tornou-se necessário compreender o conceito do divisor de tensão. Método analítico aplicado em circuitos elétricos, mais especificamente para circuitos com combinações de resistores em série. Essa técnica permite determinar a tensão por meio do resistor sem que haja a determinação da corrente do circuito. Em geral, esta técnica é aplicada para circuitos que englobem $\mathrm{N}$ resistores em série, tendo no enésimo resistor uma queda de tensão, conforme apresentado na Figura 3.

Figura 3 - Circuito Elétrico (a) Circuito divisor de tensão. (b) Divisor de tensão do ohmímetro.

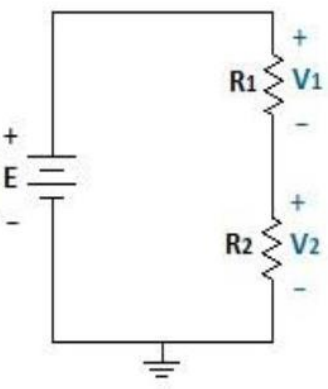

(a)

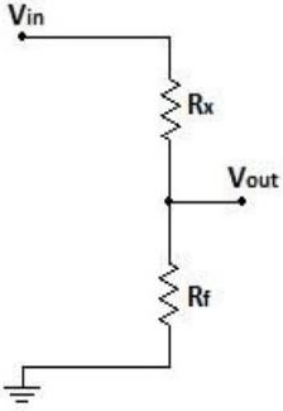

(b)

Fonte: Autoria Própria.

A tensão inicial (E) aplicada nas extremidades dos resistores subdivide-se entre os dois elementos, visto que os mesmos estão em série. De acordo com a definição do divisor de tensão, tem se a expressão para V1 ou V2 é:

$$
V_{x}=R_{x} \frac{E}{R_{e q}}
$$

Para o circuito divisor de tensão apresentado na Figura $3(b)$, tem-se o conhecimento da resistência $R_{f}$. Pode-se deduzir o valor da corrente por meio da Lei de Ohm, e ao utilizar 
a Equação 1, obter a expressão para os cálculos que determinam os valores das resistências:

$$
R_{x}=\frac{R_{f} \cdot V_{\text {out }}}{V_{\text {in }}-V_{\text {out }}}
$$

em que, $R_{x}$ é o resistor desconhecido, $R_{f}$ é o resistor de referência, $V_{i n}$ e $V_{\text {out }}$ as tensões de entrada e saída, respectivamente.

Após determinar a resistência de referência do circuito, em que a mesma implica na escala do ohmímetro, a Equação (2), demonstra que a relação $R_{x}$ e $V_{\text {out }}$ não são lineares.

Para que não haja discrepância nos valores a serem medidos, e para que mantenha uma tolerância máxima de $5 \%$ tornou-se necessário cálculos para determinação dos parâmetros. Desta forma, pode se verificar na Tabela 1 as faixas de medidas dos resistores, no qual variam de $1 \Omega$ à $40 \mathrm{M} \Omega$, com seus respectivos resistores de referência.

Tabela 1: Resistores de referência com os devidos Intervalos de medidas alcançados

\begin{tabular}{ccc}
\hline \multirow{2}{*}{ Resistor de referência $(R f)$} & \multicolumn{2}{c}{ Resistores desconhecidos $(R x)$} \\
& Mínimo & Máximo \\
\hline $47,00 \Omega$ & $1,03 \Omega$ & $2,24 \mathrm{k} \Omega$ \\
\hline $100,00 \Omega$ & $2,20 \Omega$ & $4,77 \mathrm{k} \Omega$ \\
\hline $1,00 \mathrm{k} \Omega$ & $21,96 \Omega$ & $47,76 \mathrm{k} \Omega$ \\
\hline $10,00 \mathrm{k} \Omega$ & $219,56 \Omega$ & $477,62 \mathrm{k} \Omega$ \\
\hline $100,00 \mathrm{k} \Omega$ & $2,19 \mathrm{k} \Omega$ & $4,77 \mathrm{M} \Omega$ \\
\hline $1,00 \mathrm{M} \Omega$ & $21,95 \mathrm{k} \Omega$ & $47,76 \mathrm{M} \Omega$ \\
\hline
\end{tabular}

Fonte: Autoria Própria

A Tabela 1 apresenta alguns parâmetros para o qual o ohmímetro pode operar dentro da faixa de tolerância permitida. Os valores listados abordam as resistências mínima e máxima que cada resistor de referência pode apresentar. Para aumentar a escala de medidas da ferramenta, foi adicionado novos resistores de referência.

A plataforma Arduino apresentou-se como parte da construção e execução do projeto, devido a sua praticidade e histórico na instrumentação de medidas físicas. A visualização dos dados é visualizada pelo display LCD acoplado ao projeto, conforme apresenta-se na Figura 4. 
Figura 4 - Circuito do Ohmímetro.

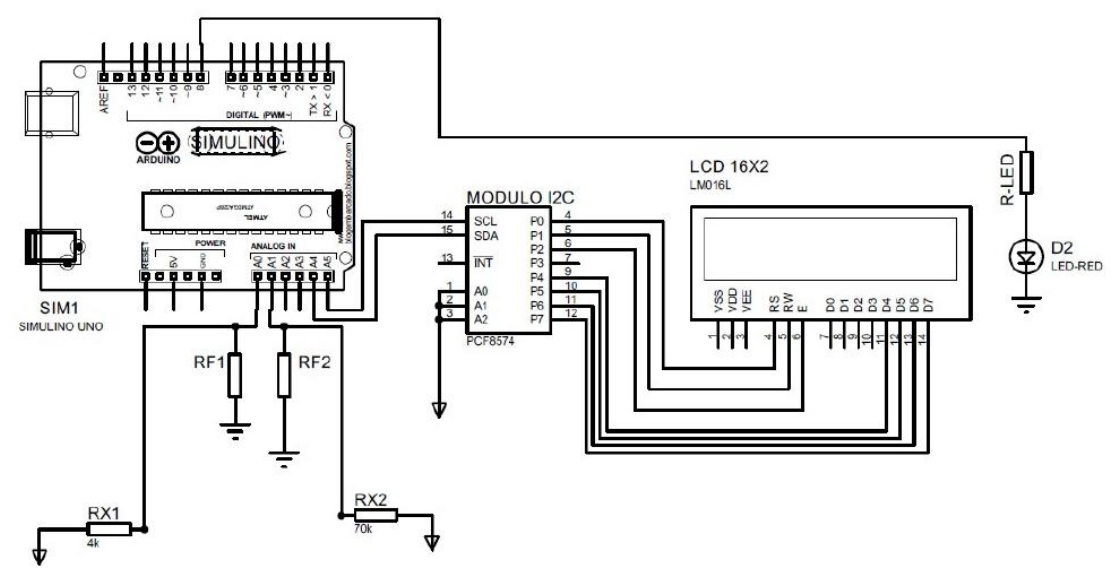

Fonte: Autoria Própria.

O desenvolvimento e implementação do projeto se resume na montagem do divisor de tensão alimentado por uma fonte de 5 Volts proveniente do Arduino. O divisor de tensão é conectado em uma das seis portas analógicas do microcontrolador, que por sua vez, digitaliza a tensão por meio de um conversor analógico-digital de 10 bits, no qual é realizado por uma combinação de 1024 bits, sendo cada bit equivalente a 4,88 milivolts de acordo a tensão de 5 Volts fornecida.

Para o modelo de ohmímetro proposto, utilizou-se dois resistores de referência, sendo respectivamente $1 \mathrm{k} \Omega$ e $10 \mathrm{k} \Omega$, conectado por duas das seis portas analógicas disponibilizadas pelo instrumento. Outros dois pinos foram destinados à conexão do módulo que conecta o Arduino ao display LCD.

A utilização do LED como meio de otimização para fins de conexão e visualização, proporcionaram uma rápida análise acerca dos resistores, avaliando se as conexões estavam corretamente. Caso o LED esteja piscando, o ohmímetro portátil estará apresentando algum tipo de mensagem que deve ser visualizada na tela LCD, conforme apresenta a Figura 5.

Figura 5 - Circuito do Ohmímetro Implementado

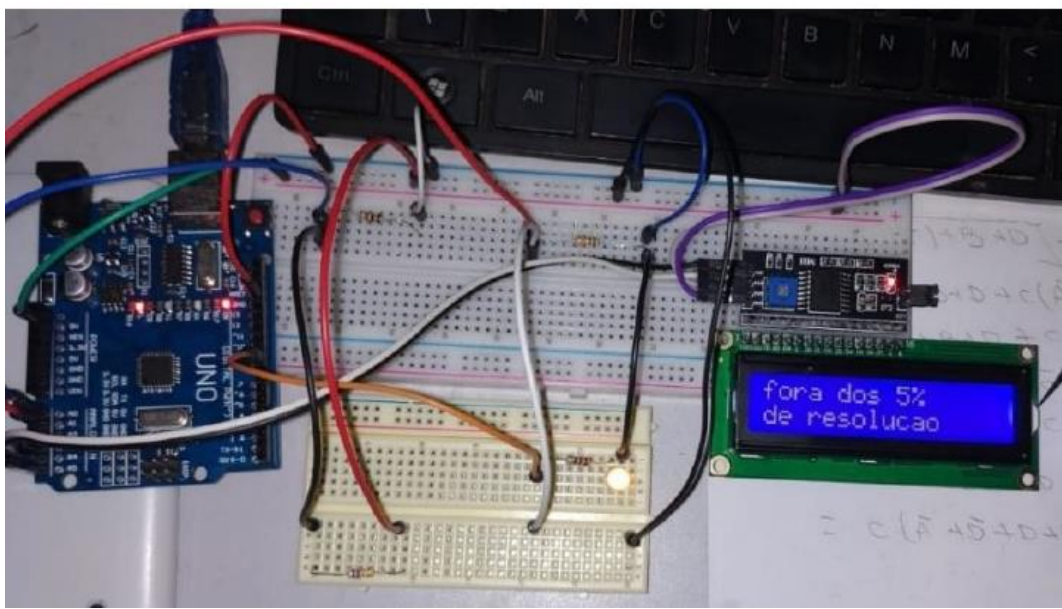

Fonte: Autoria Própria 
Em virtude de algumas limitações impostas pela plataforma Arduino, foi realizado uma análise da relação entre $R_{x}$ e $V_{\text {out }}$ na Equação (2). Foi observado no display que para as tensões de saída $\left(V_{\text {out }}\right)$ muito próximas de 0 e 5 Volts, os valores de $R_{x}$ não correspondiam às variações de tensão condizentes a realidade. Para tanto, fez se necessário limitar o Arduino, de tal forma que as variações de tensões medidas pelo instrumento retornassem aos valores das resistências dentro do intervalo de tolerância permitida.

\section{$4 \quad$ RESULTADOS E DISCUSSÕES}

Finalizada a construção e implementação do Ohmímetro, deu-se início aos testes de funcionamento.

A partir dos parâmetros, foram realizados testes e comparações dos valores obtidos no ohmímetro desenvolvido com o multímetro digital qualquer. Verificou a precisão do instrumento para diferentes valores de resistência. Na Figura 6 apresentam-se os valores dos resistores de (a) $70 \mathrm{k} \Omega$ e (b) $220 \Omega$, respectivamente, no qual pode se comprovar que 0 ohmímetro portátil identifica o resultado coerente com os multímetros tradicionais.

Figura 6 - Comparação dos valores de resistência

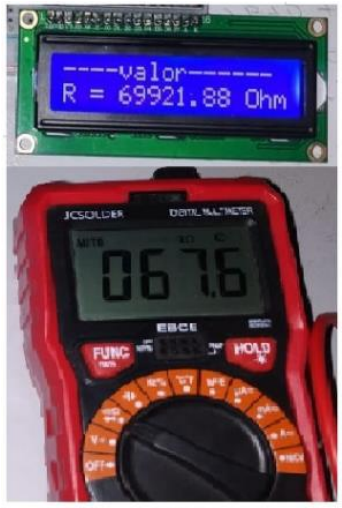

(a)

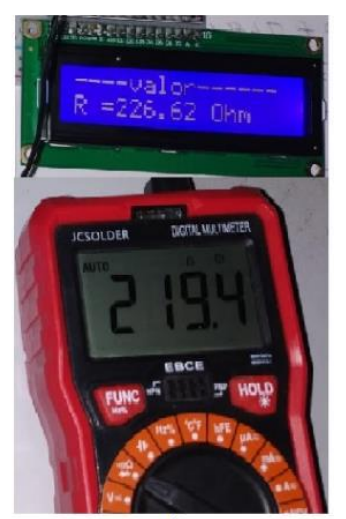

(b)

Fonte: Autoria Própria

Os resultados alcançados foram similares em ambos equipamentos, constatando sua eficácia na medição via tolerância do resistor. Em fomento à questão da conectividade do componente e a escala a ser utilizada no ato das medições, o instrumento tem como diferencial um sistema de comandos agregados ao Arduino, conforme apresenta-se na Figura 7. 
Figura 7 - Informações de conectividade fornecidas pelo Ohmímetro

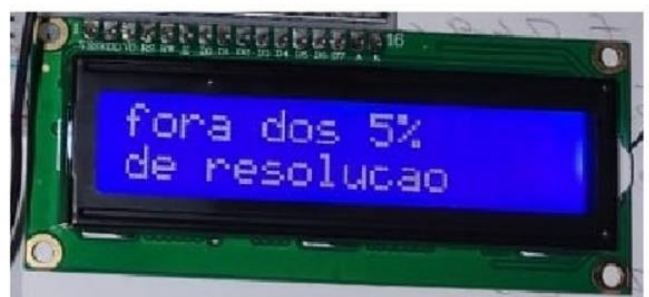

(a)

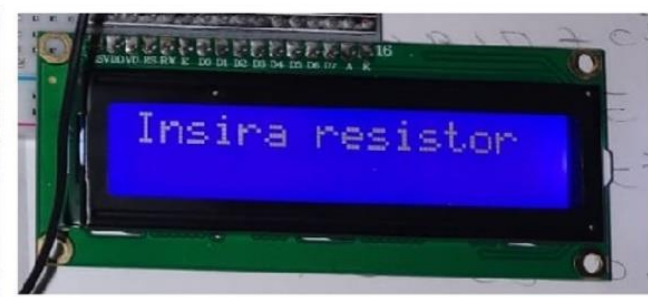

(b)

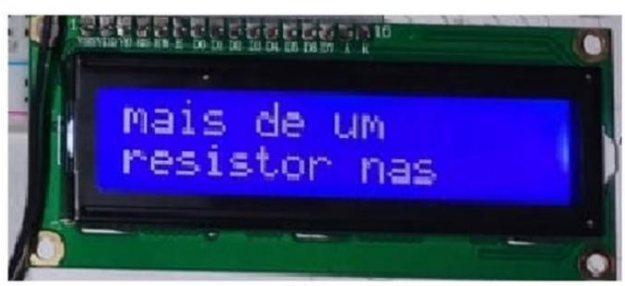

(c)

Fonte: Autoria Própria

Estes aspectos por sua vez, tornam a prática mais didática, dado que a correção de alguns dos principais equívocos encontrados na performance da ferramenta está direcionada ao próprio instrumento. Para caráter de manuseio, o display informa ao usuário o estado de funcionamento do equipamento por meio de frases como, "insira um resistor", "fora dos 5\% de resolução", "mais de um resistor". E então o leitor verifica se de fato suas medições estão coerentes com a realidade. A programação realizada para identificação e leitura dos resistores é apresentada na Figura 8.

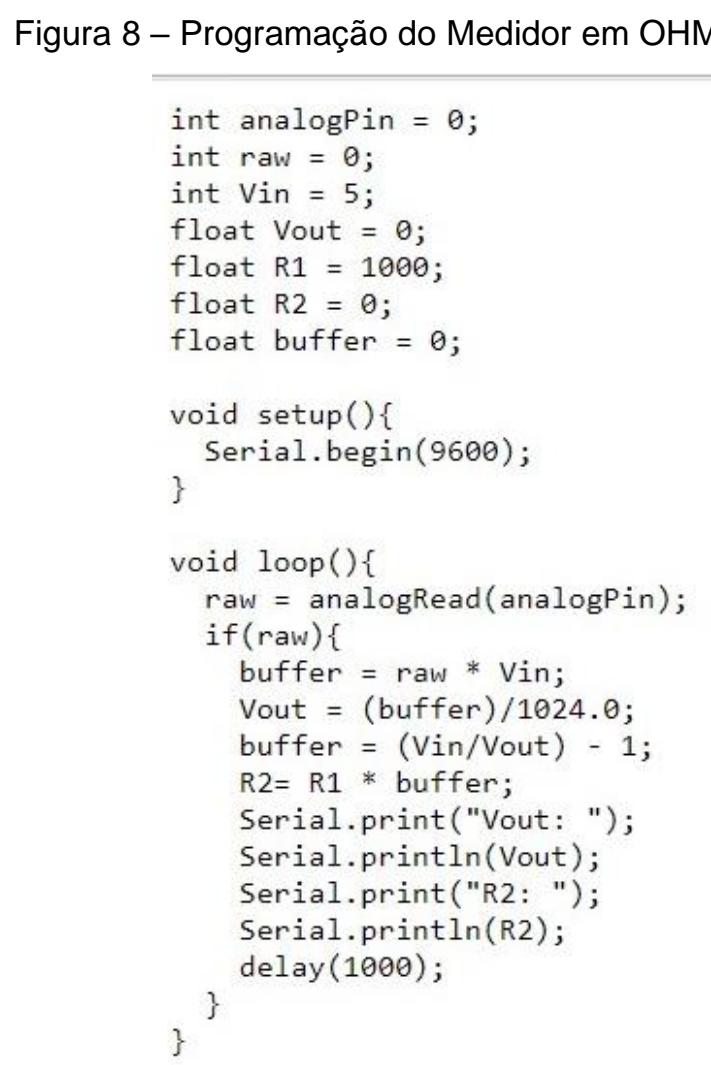

Fonte: Autoria Própria 
Ao alterar o valor da resistência conhecida no circuito, o programa também será alterado para reconhecimento do sistema. Como um multímetro convencional, este circuito possui algumas faixas para medir a resistência, eis a parte da programação juntamente com a tela de LCD para informar ao usuário. Se você tentar medir uma resistência de baixo valor na faixa de mega ohms do multímetro, certamente obterá valores de erro.

Também indicar o valor limite no programa, uma vez que ao ultrapassar o limite predeterminado, o Arduino informará ao usuário por meio da tela de LCD

\section{CONCLUSÃO}

Este artigo teve como propósito construir um instrumento de fácil manuseio as mais diversas modalidades de ensino, seja no nível técnico ou superior, o que possibilita reprodução para as inúmeras aplicações, abrindo um emaranhado de possibilidades de projetos.

Despertar o interesse de estudantes, estimulando e facilitando a iniciativa de novos equipamentos autônomos que desfrutem do fato de materiais específicos mudarem a resistência de acordo com condições físicas como luminosidade, umidade, pressão e temperatura, dando ideia de como desenvolver sensores, no qual a medida de resistência torna-se fundamental.

Com praticidade e mobilidade o Arduino foi o componente mais indicado para o projeto, pois é de baixo custo, simples manuseio e essencial para introduzir as mais diversas práticas, tornando importante para familiarização com instrumentos de medida elétricas, englobado pela programação e circuitos de maneira simples e acessível.

Ao desfrutar de um microcontrolador para realização dos cálculos de comparação analógico/digital com portabilidade e confiabilidade, traz praticidade para os usuários que não mais necessitam de aparelhos que na maioria das vezes para os estudantes só estão disponíveis em laboratórios.

O fato de não necessitar usar ponta de prova na interação com o usuário, apresentou ser um dos desafios encontrados, pois o aparelho apesar de manter a resolução fixa tem mudanças abruptas nos valores de resistências no momento de inserção ou troca de resistores, ocorrências que acontecem também ocasionalmente durante o uso, diante do possível mal contado entre jumpers e protoboard.

Conclui-se, portanto, que o um equipamento se tornou valioso e interessante para quaisquer classificações de usuários que venham utilizá-lo, seja ele, aluno do ensino médio, técnico ou superior.

\section{REFERÊNCIAS}

ALBERTAZZI Jr. G. A., Sousa R. de A., Científica e Industrial Fundamentos de Metrologia, Editora Manole: 1a Ed., 2009

ALEXANDER, Charles K; SADIKU, Matthew NO. Fundamentos de circuitos elétricos. AMGH Editora, 2013. José, 2012.

BOYLESTAD, R. L. Introdução à análise de circuitos elétricos. Tradução de 
DO NASCIMENTO, Bruno. Medidor de grandezas elétricas com acesso remoto. Revista Eletrônica de Engenharia Elétrica e Engenharia Mecânica, v. 1, n. 2, p. 42-53, 2020.

GASPAR, A., \& C., M. I. (2005). Atividades experimentais de demonstrações em sala de aula: Uma análise segundo o referencial da teoria de Vygotsky. UNESPSP.

\section{DEVELOPMENT OF A PORTABLE AND LOW COST HOMIMETER}

Abstract: This article presents the development of a low cost, portable microcontrolled ohmmeter implemented in the discipline of electrical circuits $I$. The work was started with the aim of seeking a solution to meet the practical demands at home, given the situation of isolation and impossibility of go to the laboratories to perform practices, given that precision and security in data acquisition, are some of the essential characteristics in the handling of digital instruments for the electrical engineering course. The development used interdisciplinary contents, starting from the understanding and principle of operation of a multimeter, the programming of a microcontroller was done by Arduíno, for the treatment of data. Given the essential techniques and thus establishing a concise and objective practice, it was vital and necessary to understand the basics in circuit analysis, knowledge in programming language and fundamental physical components, such as resistors, jumpers and protoboards. In addition to the description of the components, materials and methods used for the development, the work also presents some practical tests. It is believed that the resources shown here, associated with theoretical classes, provide a more favorable environment for learning, making the student have curiosity and pleasure in learning, being a valuable didactic complementation tool.

Keywords: Arduino, Voltage Divider, Programming, Resistors 